\title{
The importance of measuring the solvent quality of unfolded proteins.
}

Tobin R Sosnick $^{\mathrm{a} *}$, Joshua A Riback ${ }^{\mathrm{b}}$, Micayla A Bowman ${ }^{\mathrm{c}}$, Adam M Zmyslowski ${ }^{\mathrm{a}}$, Anabel Rodriguez ${ }^{\mathrm{c}}$, Kevin W Plaxco ${ }^{\mathrm{d}}$, Patricia L Clark ${ }^{\mathrm{c*}}$

${ }^{a}$ Dept. of Biochemistry and Molecular Biology, University of Chicago, trsosnic@uchicago.edu

${ }^{\mathrm{b}}$ Graduate Program in Biophysical Sciences, University of Chicago

'Dept. of Chemistry and Biochemistry, University of Notre Dame, pclark1@nd.edu

${ }^{\mathrm{d} D e p t . ~ o f ~ C h e m i s t r y ~ a n d ~ B i o c h e m i s t r y, ~ U n i v e r s i t y ~ o f ~ C a l i f o r n i a, ~ S a n t a ~ B a r b a r a ~}$

The conformational properties of unfolded and intrinsically disordered proteins are relevant to folding and biothermodynamics as well as biological processes including transport, turn-over and liquid-liquied phase separation. However, the characterization of disordered states is challenging, in part due to a lack of accurate models and the difficulty of interpreting experimental results. We developed a method

to extract the dimensions of disordered states and the solvent quality $\left(v\right.$ in $\left.R_{g} \propto N_{\text {res }}{ }^{v}\right)$ from a single SAXS measurement. ${ }^{1-2}$ We find that even relatively hydrophobic IDPs can remain nearly as expanded as the chemically denatured ensemble with $v$ value between $0.52-0.55$, above the random walk value of $1 / 2$. Hence, water should be considered to be a good solvent for unfolded states of foldable sequences and an early collapse step is not obligatory in the folding process. While advances in SAXS and FRET analysis has narrowed the difference in their views of the dimensions of unfolded states, we show that fluorophore-based interactions along with improved analysis procedures can explain the preponderance of existing data. ${ }^{3}$ We examine the robustness of these results and find that a slight reshuffling of the sequence to create local hydrophobic or charge regions can induce collapse and aggregation, and affect protein secretion. These results suggest that sequence patterns are under selection to be well-mixed with $v>1 / 2$ in part to minimize protein misfolding, aggregation and other undesired associations. The requirement to be well-mixed also puts constraints on allowed protein architectures.

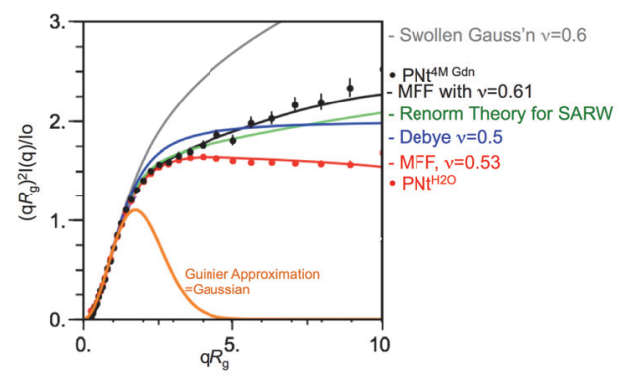

References.

1. Riback, J. A.; Bowman, M. A.; Zmyslowski, A. M.; Knoverek, C. R.; Jumper, J. M.; Hinshaw, J. R.; Kaye, E. B.; Freed, K. F.; Clark, P. L.; Sosnick, T. R., Innovative scattering analysis shows that hydrophobic disordered proteins are expanded in water. Science 2017, 358, 238.

2. Riback, J. A.; Bowman, M. A.; Zmyslowski, A.; Knoverek, C. R.; Jumper, J.; Kaye, E. B.; Freed, K. F.; Clark, P. L.; Sosnick, T. R., Response to Comment on "Innovative scattering analysis shows that hydrophobic disordered proteins are expanded in water". Science 2018, 361 (6405).

3. Riback, J. A.; Bowman, M. A.; Zmyslowski, A. M.; Plaxco, K. W.; Clark, P. L.; Sosnick, T. R., Commonly used FRET fluorophores promote collapse of an otherwise disordered protein. Proc Natl AcadSci USA 2019, $116,8889$. 